Research Article

\title{
Offline Signature Authentication Algorithm Based on the Fuzzy Set
}

\author{
Shi Qiu $\mathbb{D}^{1},{ }^{1}$ Fengchang Fei $\mathbb{D}{ }^{2}$ and Ying $\mathrm{Cui}^{3}$ \\ ${ }^{1}$ Key Laboratory of Spectral Imaging Technology CAS, Xi'an Institute of Optics and Precision Mechanics, \\ Chinese Academy of Sciences, Xi'an 710119, China \\ ${ }^{2}$ College of Modern Economics and Management, Jiangxi University of Finance and Economic, Nanchang 330077, China \\ ${ }^{3}$ College of Equipment Management and Support, Engineering University of PAP, Xi'an 710086, China \\ Correspondence should be addressed to Fengchang Fei; ffcbox@163.com
}

Received 28 January 2021; Revised 26 February 2021; Accepted 10 March 2021; Published 19 March 2021

Academic Editor: Chenxi Huang

Copyright (c) 2021 Shi Qiu et al. This is an open access article distributed under the Creative Commons Attribution License, which permits unrestricted use, distribution, and reproduction in any medium, provided the original work is properly cited.

\begin{abstract}
There exists a problem that it is difficult to identify the authenticity of offline signatures. Firstly, a segmentation model is established based on the theory of fuzzy sets to extract signatures completely. Secondly, statistical shape model (SSM) and variance distance discretization of intraclass signatures are introduced for stability analysis and quantification. Finally, multilayer classifiers are constructed to realize signature authentication. The algorithm has low false detection rate and short authentication time.
\end{abstract}

\section{Introduction}

Handwritten signature authentication plays an important role in many fields [1]. Handwritten signature is a behavioral feature acquired for a period of time, and it belongs to the individual behavioral biological feature. With the rapid development of the information industry and service industry, signature authentication has attracted wide attention and has been widely used in many fields, such as financial security and identity authentication.

Signature authentication can be classified into offline and online. Offline authentication relies on static image information such as shape and texture [2]. Online authentication contains more information than offline authentication. It can be judged by dynamic information in the signature process, such as writing order, pen pressure, and writing speed [3]. Research studies on online authentication have been carried out for a long time, and good results have been achieved. Owing to the lack of dynamic signature information and available information in most cases, offline signature authentication is more difficult, but the demand for offline authentication is huge in practical applications. Offline signature authentication based on the image usually includes preprocessing, feature extraction, and signature authentication. Preprocessing mainly includes gray histogram enhancement [4], signature extraction [5], edge information enhancement [6], and image size normalization [7]. Feature extraction includes gradient [8], SIFT [9], posetoriented grid features [10], entropy [11], biological features [12], HOG features [13], histogram [14], LBP [15], and wavelet [16]. Signature authentication mainly includes SVM [17], KNN [18], neural network [19], deep learning [20], hidden Markov model [21], and artificial immune system [22].

Research studies on offline signature authentication are mainly focused on the following: (1) how can the mechanism of separating the signature from the background be constructed to extract signature information completely? (2) How can effective signature image features be extracted? (3) How can a perfect authentication mechanism be established? In order to solve these problems, we are going to construct a robust offline signature authentication model: (1) a signature extraction framework based on the fuzzy set is proposed. (2) A signature stability authentication mechanism is constructed to select stable features. (3) According to the stability of the signature, a multilevel authentication system is established to realize the signature authentication accurately. 


\section{Algorithm}

Signatures can be affected by emotions, posture, fatigue, and other factors from psychological analysis, which can lead to fluctuations in my signature to a certain extent [23]. These differences can challenge signature authentication. Figure 1 shows the overlapping images of five signatures from two signers with different stabilities. It appears that the stability of Figure $1(\mathrm{a})$ is stronger than that of Figure 1(b). The stability of signature authentication classifiers can affect the performance of signature authentication classifiers.

Thus, we construct a multilevel signature authentication system. The flowchart is shown in Figure 2. (1) Signature images can be extracted to preprocess based on the theory of fuzzy sets. (2) SSM model and variance distance algorithm can be constructed to analyze the stability of quantized signatures. All signature samples can be divided into stability and instability. (3) For stable samples, we combine with the advantages of the hybrid extreme learning machine and sparse representation to construct a HCSV classifier for authentication. For unstable samples, template matching is used to authenticate them.

\subsection{Signature Extraction Algorithm Based on the Fuzzy Set.} Complete signature extraction is the precondition of subsequent operations. Different intensities in the process of signature result in different gray values on the image. So, it is difficult to extract it completely. Therefore, we introduce the theory of fuzzy sets to analyze it. Interval type-2 fuzzy set (IT2FS) can effectively deal with data uncertainty. It has attracted wide attention of scholars owing to the superiority of the processing effect. Gonzalez et al. [24] used IT2FS to extract the image boundary. Shi et al. [25] established a model based on the IT2FS to achieve specific shape segmentation. Dhar and Kundu [26] fused IT2FS and Bat to realize segmentation threshold calculation. How we measure the uncertain information represented by the IT2FS is the focus of current research.

We propose a new method for calculating interval twodimensional fuzzy entropy. On the basis of satisfying the definition of the fuzzy set and considering the influence of membership degree's fuzziness and mean value on entropy, the uncertainty information represented by the IT2FS is measured comprehensively and objectively.

Four axioms should be satisfied for the classical interval two-type fuzzy entropy $E(A)$ :

$\mathrm{R} 1$ : if $A$ is a nonfuzzy set, then $E(A)=0$

R2: $E(A) \leq E\left(A^{c}\right), \quad \forall A \in I T 2 F S, A^{c}$ is the complement of $A$

R3: $E(A) \leq E(B)$ if fuzzy $B$ is more than $A$ : when $\bar{B}\left(x_{i}\right)+\underline{B}\left(x_{i}\right) \leq 1, \underline{A}\left(x_{i}\right) \leq \underline{B}\left(x_{i}\right) ; \bar{A}\left(x_{i}\right) \leq \bar{B}\left(x_{i}\right)$; when $\bar{B}\left(x_{i}\right)+\underline{B}\left(x_{i}\right)>1, \underline{A}\left(x_{i}\right)>\underline{B}\left(x_{i}\right) ; \bar{A}\left(x_{i}\right)>\bar{B}\left(x_{i}\right)$

R4: when $\underline{A}\left(x_{i}\right)+\bar{A}\left(x_{i}\right)=1, E(A)=1$

$\bar{A}\left(x_{i}\right)$ and $\underline{A}\left(x_{i}\right)$ are the upper approximation set and the lower approximation set of $A$.
Burillo and Bustince [27] established interval type-2 fuzzy entropy as

$$
E(A)=\frac{1}{N} \sum_{i=1}^{N}\left(\bar{A}\left(x_{i}\right)-\underline{A}\left(x_{i}\right)\right)
$$

Fully considering the fuzziness of membership degree, the difference between upper and lower bounds of membership degree is proportional to the value of entropy, but the influence of the mean value of membership degree is not considered, and R3 and R4 are not satisfied.

Zeng and $\mathrm{Li}$ [28] established interval type-2 fuzzy entropy as

$$
E(A)=1-\frac{1}{N} \sum_{i=1}^{N}\left|\bar{A}\left(x_{i}\right)+\underline{A}\left(x_{i}\right)-1\right| .
$$

Four axioms are satisfied, and the influence of membership degree mean is fully considered, but the influence of the fuzziness of membership degree itself about interval type- 2 fuzzy entropy is not considered.

We revise the above two interval type-2 fuzzy entropies:

$$
E(A)=\frac{N-\sum_{i=1}^{N}\left|\bar{A}\left(x_{i}\right)+\underline{A}\left(x_{i}\right)-1\right|\left(1-\bar{A}\left(x_{i}\right)+\underline{A}\left(x_{i}\right)\right)}{N+\sum_{i=1}^{N}\left|\bar{A}\left(x_{i}\right)+\underline{A}\left(x_{i}\right)-1\right|\left(1-\bar{A}\left(x_{i}\right)+\underline{A}\left(x_{i}\right)\right)} .
$$

The influence of membership degree's fuzziness on interval type-2 fuzzy entropy is considered while satisfying the interval type- 2 fuzzy entropy theorem, and IT2FS is used to measure the uncertainty information comprehensively. The corresponding gray value at the minimum of this function is used to realize the accurate image segmentation.

In order to ensure the unity of the objective function, we normalize the real handwritten signature sample $\left\{X_{i}=1, \ldots N\right\}$. The signature center line is made as the center, and the resolution of the signature image is unified to $256 \times 128$ by the nearest neighbor interpolation algorithm. On this basis, the signature center line is extracted. According to the characteristics of the signature, it should satisfy the following: (1) the center line must be continuous, and the handwriting cannot break as far as possible. (2) Center line preserves the signature framework. (3) The cross section of handwriting should be as little distorted as possible. In this paper, the algorithm proposed in [29] is used to extract the center line.

2.2. The Stability Model. The analysis of stability is the premise of signature authentication, which can be divided into signature stability and feature stability. Different algorithms are used to authenticate it by quantization stability. For signature stability and feature stability, we use the following methods to authenticate the signature stability.

Calculate the average model of $N$ true signature features:

$$
F=\frac{1}{N} \sum_{i=1}^{N} F_{i}, \quad\left\{F_{i}=d_{i j} \mid i=1, \ldots, N ; j=1, \ldots, D\right\} .
$$

Calculate the deviation between each real sample and the average sample: 


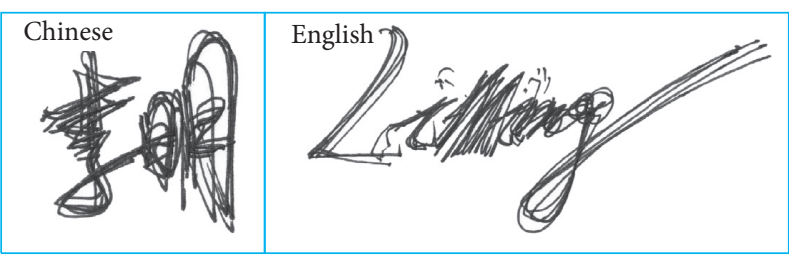

(a)

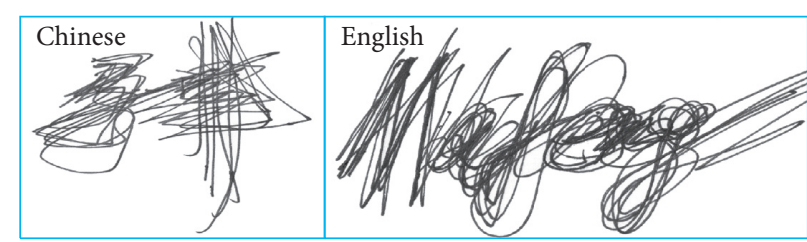

(b)

Figure 1: Signature sample groups. (a) Data 1. (b) Data 2.



Figure 2: Algorithm flowchart.

$$
\mathrm{d} F_{i}=F_{i}-\bar{F}
$$

Calculate the covariance matrix:

$$
S=\frac{1}{N} \sum_{i=1}^{N} \mathrm{~d} F_{i} \mathrm{~d} F_{i}^{T}
$$

Calculate the covariance matrix of eigenvalue $\lambda_{k}$ and eigenvector $\Phi_{k}$ :

$$
S \Phi_{k}=\lambda_{k} \Phi_{k}, \quad \lambda_{k} \geq \lambda_{k+1}, \Phi_{k}^{T} \Phi_{k}=1,(k=1, \ldots, 2 n),
$$

where $\Phi_{k}$ represents the main changes of the real signature shape model. The larger the value of $\lambda_{k}$ is, the more obvious the change of the shape model of the real signature is. We choose $\left\{\lambda_{1}, \ldots \lambda_{t}\right\},\left\{\Phi_{1}, \ldots \Phi_{t}\right\}$ to approximate the change of signature samples and reduce the number of signatures.

Let $\lambda_{T}=\sum_{k=1}^{2 D} \lambda_{k} ; \lambda_{k} / \lambda_{T}$ is the contribution rate of the $k$-th feature. The selection of $t$ should satisfy the following constraints:

$$
\left(\frac{\left[\sum_{k=1}^{t} \lambda_{k}\right]}{\lambda_{T}}\right)>\alpha, \quad 0 \leq \alpha \leq 1
$$

Calculate the weight coefficient of the main deformation parameters $b_{k}$ :

$$
F \approx \bar{F}+\sum_{k=1}^{t} b_{k} \Phi_{k}, \quad-m \sqrt{\lambda_{k}} \leq b_{k} \leq m \sqrt{\lambda_{k}}, \quad m=2,3 .
$$

When SSM [30] is used to analyze the signature and feature stability, it is necessary to quantify its stability. We quantify the stability of signatures by using the square difference dispersion of the deformation parameter $b_{k} \Phi_{k}$. Given $\left\{X_{i} \mid i=1, \ldots N\right\}$ is the true set of signature images, the mean value of class $M_{p}$ and intraclass variance $S_{p}$ can be calculated:

$$
\begin{aligned}
M_{p} & =\frac{\sum_{i=1}^{N} b_{k} \Phi_{k}}{N}, \\
S_{p} & =\sum_{k=1}^{N}\left(b_{k} \Phi_{k}-M_{p}\right)^{2} .
\end{aligned}
$$

The intraclass variance distance dispersion $\mathrm{SD}_{k}$ of each signer can be obtained for stability analysis:

$$
\left\{\begin{array}{l}
\mathrm{SD}_{k}=\left|S_{k}^{2}-M_{s}\right|, \quad k=1, \ldots, N, \\
M_{s}=\frac{\left(\sum_{k=1}^{N} S_{k}^{2}\right)}{N} .
\end{array}\right.
$$

2.3. Signature Authentication. According to different signature stabilities, different authentication algorithms are constructed. For stable signatures, handwritten signature needs to be classified by efficient algorithms because it has few sample data. The ELM (extreme learning machine) algorithm [31] has the advantages of less training parameters and faster classification speed and is suitable for small sample datasets. The SRC (sparse representation classifier) [32] can sparsely represent features, reduce the storage of signature data, omit the pretraining stage of signature authentication, and reduce the complexity of the algorithm.

In order to fully consider the effectiveness for a given period of time, we combine the advantages of the ELM and SRC to design a hierarchical offline handwritten Chinese signature authentication scheme, as shown in Figure 3. Firstly, the ELM based on the deterministic threshold of signature authentication is used to realize the first-level rough classification of the signature samples, which are 




Figure 3: The flowchart of signature authentication.

ready to be tested. Then, a sparse representation dictionary for the false signature and real signature is designed and implemented. The signature authentication that cannot be identified in the first step is identified by solving the sparse coefficients.

First-level classification: in order to solve the classification problem of authentic and false data with less similarity between signature data, we design an ELM structure as follows: the input layer and the hidden layer of the signature sample data and the output layer of the authentication certainty of the signature sample data are used to obtain the authenticity of the signatures, which are ready to be tested.

There is a single hidden layer neural network for the signature image with $N$ hidden layer nodes:

$$
V=\sum_{i=1}^{N} \beta_{i} g\left(W_{i} \cdot X_{j}+b_{i}\right) \quad j=1, \ldots N,
$$

where $V$ is the determination of the authentication signatures; $\beta_{i}$ is the output weight; $W_{i}$ is the input weight; $\bullet$ is the inner product; $b_{i}$ is the offset of the $i$-th hidden layer unit; and the threshold $V_{T}$ is set to determine the accuracy of the results. When $V>V^{T}$, it shows that the classification of the algorithm is reliable and can output the results directly. On the contrary, it shows that the classification reliability of the algorithm is low, and the samples need to be sent to the next level classifier for further discrimination.

Second-level classification: in order to solve the problem that the first-level classifier cannot effectively identify the authentication of samples with high similarity, a supercomplete signature dictionary is designed. All signatures of the same writer are collected as samples and classified into two categories: $C \in\{0,1\} .0$ denotes the fake signature samples, and 1 denotes the real signature samples. Then, all samples can be expressed as $D=\left[A_{0}, A_{1}\right] . A_{i}$ represents the matrix composed of $n_{i}$ samples arranged in the $i$-th class. The test sample of the signature can be sparsely represented as

$$
y=D X_{o} \quad X_{o}=\left[0, \ldots 0, a_{11}, a_{12} \ldots, a_{1 n_{i}}\right]
$$

where the nonzero term corresponding to $X_{o}$ belongs to the real signature. The problem of solving the sparse coefficients of the linear combination of models is the classification of signature authentication.

The solution of sparse coefficients of the signature classification is a process of reconstructing test signature samples by linear combination of all atoms in the constructed supercomplete signature dictionary. The problem of solving $\mathrm{X}$ by the model can be transformed into

$$
x=\underset{x}{\arg \min }\left\|x_{0}\right\|
$$

where $\|\cdot\|$ represents the $L_{0}$-norm operation. Equation (15) is transformed into an NP-hard problem because the constructed $D$ is a supercomplete dictionary of true and false signatures.

At this time, $L_{1}$ is easier to solve than the $L_{0}$-norm, and $L_{1}$ is more sparse than the $L_{2}$-norm. Therefore, we use the $L_{1}$-norm to solve the classification problem of authentic and false signatures and then use the minimum residual method in the sparse classification to authenticate signatures.

For the unstable signature model, we use classical $K$ means clustering to cluster discrete unstable signature samples into templates and then use clustering centers as template matching.

Input $M$ sample datasets for $X=\left\{X_{i} \mid i=1, \ldots, M\right\}$, and input $K$-clustering categories $C=\left\{C_{j} \mid j=1, \ldots, k\right\}$, where $C_{j}=1 / n_{j} \sum_{x \in C} x$.

Energy function $E=\sum_{i=1}^{K} \sum_{J=1}^{n} d\left(x_{j}, c_{i}\right)$ is constant by iteration calculation, where $d\left(x_{j}, c_{i}\right)=\sqrt{\left(x_{j}-c_{i}\right)^{T}\left(x_{j}-c_{i}\right)}$.

\section{Experiment and Result Analysis}

We construct English and Chinese databases, which are composed of the number of real signatures, the number of skilled fake signatures, and the number of unskilled fake signatures. Database 1 is from [33]. In order to verify the algorithm performance, we collect practical data to construct databases 2 and 3. The database is shown in Table 1. The proposed algorithm is written by $\mathrm{VC}++$ software and WIN7 operation platform. The data ratio of the test set and training set is $1: 1$. 
TABLe 1: Database composition.

\begin{tabular}{|c|c|c|c|c|c|}
\hline & \multirow{2}{*}{ Database type } & \multirow{2}{*}{ People no. } & \multirow{2}{*}{ Actual signature no. } & \multicolumn{2}{|c|}{ Forged signature no. } \\
\hline & & & & Skilled & Unskilled \\
\hline 1 & English & 24 & 480 & 240 & 240 \\
\hline 2 & English & 20 & 300 & 150 & 150 \\
\hline 3 & Chinese & 11 & 440 & 220 & 220 \\
\hline
\end{tabular}

TABLE 2: The effect of signature extraction algorithms.

\begin{tabular}{llr}
\hline Algorithm & $M$ & The running time (S) \\
\hline Otsu & 86 & 0.03 \\
Continuity & 90 & 0.12 \\
EIT2FS & 94 & 0.15 \\
Ours & 96 & 0.10 \\
\hline
\end{tabular}

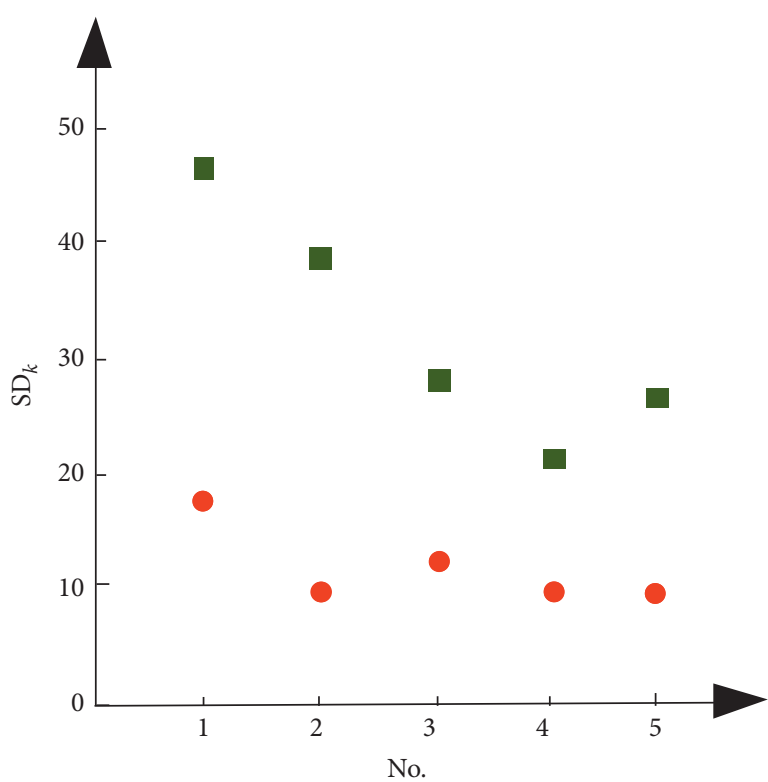

Signer 1

Signer 2

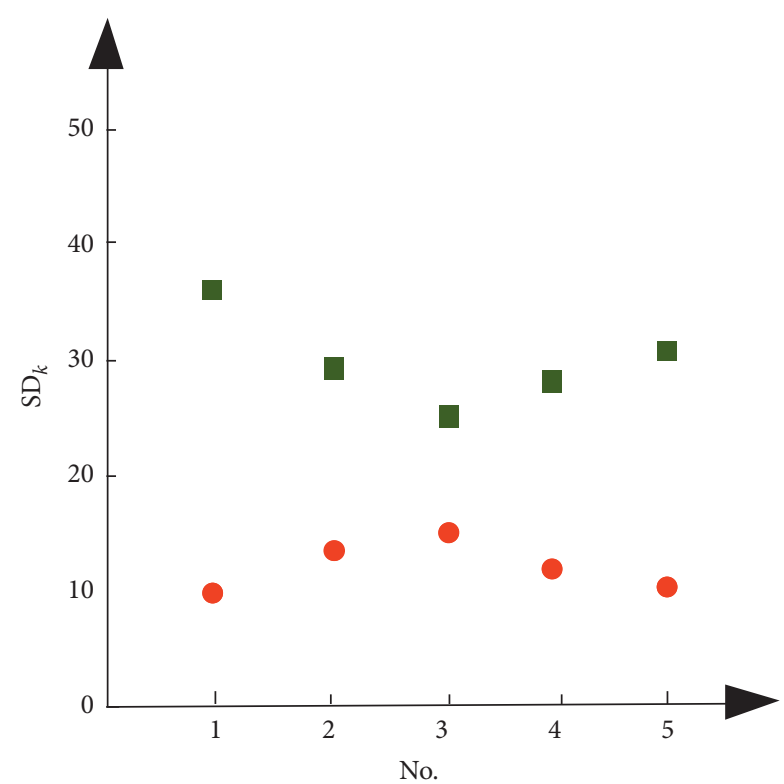

Signer 1

Signer 2

(a)

(b)

FIGURE 4: Signature stability distribution. (a) Chinese signature stability distribution map. (b) English signature stability distribution map.

The proposed algorithm is related to the quantity and quality of the dataset. Currently, only Chinese and English signatures have been tested, and some results have been achieved. The proposed algorithm can be applied into other languages.

3.1. The Comparison of Signature Extraction Algorithms. In order to assess the algorithm performance, we introduce

$$
M(A, B)=\frac{S(A \cap B)}{S(A \cup B)} \times 100 \%,
$$

where $M$ is the area overlap degree; $A$ is the gold standard for signatures extracted manually through vision; $B$ is the result of algorithm extraction; and $S($.$) is the number of pixels$ representing the corresponding region. The bigger the $M$ value is, the better the segmentation effect is.

According to Table 2, the signature can be extracted by all the algorithms involved. Otsu algorithm [5] establishes segmentation threshold according to the distribution of the gray histogram, which can quickly realize signature extraction. Continuity algorithm [34] extracts signatures one by one according to the continuity of signatures. EIT2FS algorithm [26] establishes a model with full consideration of noise interference based on the IT2FS. Our algorithm improves the interval type- 2 fuzzy entropy. It satisfies the interval type- 2 fuzzy entropy theorem and considers the influence of the membership degree's fuzziness on the interval type- 2 fuzzy entropy so that IT2FS can represent the uncertainty 
TABLE 3: The algorithm extracts the signature effect.

\begin{tabular}{cccccccc}
\hline & & & Data (\%) & & \\
& \multirow{2}{*}{ Method } & 1 & 2 & 3 & 4 & 5 & Average \\
\hline \multirow{2}{*}{ FRR } & 1 & 8.2 & 7.1 & 6.5 & 7.6 & 6.9 & 7.26 \\
& 2 & 12.3 & 11.3 & 13.5 & 10.5 & 14.2 & 7.36 \\
\hline \multirow{2}{*}{ FAR } & 1 & 6.1 & 7.5 & 6.0 & 8.1 & 7.1 \\
& 2 & 9.6 & 8.9 & 10.2 & 12.5 & 11.5 & 10.54 \\
\hline
\end{tabular}

TABLE 4: Performance comparison of signature authentication algorithms.

\begin{tabular}{lccc}
\hline Algorithm & Average FRR & Average FRR & Average FRR + FRR \\
\hline SVM & 15.2 & 8.0 & 23.2 \\
KNN & 13.1 & 8.7 & 21.8 \\
SRC & 11.3 & 6.2 & 17.5 \\
ELM & 9.2 & 4.6 & 13.8 \\
BP & 7.7 & 5.2 & 12.9 \\
Ours & 6.3 & 4.8 & 11.1 \\
\hline
\end{tabular}

information more comprehensively. Although the extraction time is longer than the algorithm in [5], the $M$ value is the highest.

3.2. The Stability Analysis. We use the SSM algorithm and variance distance dispersion to measure the signature stability according to the sample. As shown in Figure $4, \mathrm{SD}_{k}$ of signer 1 is smaller than that of signer 2 , and the fluctuation is small. It can be concluded that the stability of signer 1 is higher than that of signer 2 .

In order to test the effectiveness of signature stability analysis, we introduce acceptance rate (FAR) and rejection rate (FRR) as evaluation criteria:

$$
\begin{aligned}
& \mathrm{FAR}=\frac{\mathrm{FWA}}{N} \times 100 \%, \\
& \mathrm{FRR}=\frac{\mathrm{FGR}}{N} \times 100 \% .
\end{aligned}
$$

We have compared the signature sets of five signers. FWA is the number of forged signatures wrongly accepted. FRR is the number of genuine signatures wrongly rejected. $N$ is the total samples. We use KNN (method 1) separately and KNN (method 2) clustering based on stability analysis. The effect is shown in Table 3. It can be seen that the clustering effect of the algorithm can be improved by the data of stability analysis.

3.3. Signature Authentication. Because the signature samples are restricted by privacy and other factors, it is impossible to obtain a large number of samples. Thus, we compare our algorithm with the mainstream algorithms in all datasets on the premise of small sample size as shown in Table 4. The SVM algorithm [17] requires a large number of data samples, and the authentication effect is not good under the condition of small samples. Compared with the SVM, KNN algorithm [18] has better data requirements, but its complexity is higher. SRC [32] extracts features sparsely and better. ELM algorithm [31] realizes fast classification for small samples. BP algorithm [19] can construct a neural network structure to realize signature authenticity identification. The proposed algorithm establishes a two-level classifier, which identifies the authentication one by one according to its stability, and achieves the best result.

\section{Conclusion}

Owing to the problem that it is difficult to authenticate offline signatures, an offline signature authentication method is proposed, which is suitable for multilanguages. Firstly, a segmentation model is constructed based on the theory of fuzzy set to extract the signature completely. Then, stability analysis and quantification model are proposed to identify the stability of data and features. Finally, a two-level classifier structure is constructed to authenticate signatures one by one. In the future, we will build databases in different languages, analyze the characteristics of different languages, and realize fast signature recognition.

\section{Data Availability}

All the used data are included within the paper. Previously reported data were used to support this study and are available at https://cedar.buffalo.edu/NIJ/data/. These prior studies (and datasets) are cited at relevant places within the text as reference [33].

\section{Conflicts of Interest}

The authors declare that they have no conflicts of interest.

\section{Acknowledgments}

This work was supported by the Postdoctoral Science Foundation of China (Grant no. 2020M682144), the Science and Technology Fund of Jiangxi Education Department (Grant no. GJJ161552), the Natural Science Foundation of Jiangxi Science and Technology Department (Grant no. 20171BAB202032), Basic Research Project of Engineering University of PAP (Grant no. WJY202145), and the Open 
Project Program of the State Key Lab of CAD and CG (Grant no. A2026), Zhejiang University.

\section{References}

[1] R. Tolosana, R. Vera-Rodriguez, J. Fierrez, and J. OrtegaGarcia, "Exploring recurrent neural networks for on-line handwritten signature biometrics," IEEE Access, vol. 6, pp. 5128-5138, 2018.

[2] M. Liwicki, K. Riesen, R. Ingold et al., "Offline signature verification by combining graph edit distance and triplet networks," in Proceedings of the Joint IAPR International Workshop, S + SSPR 2018, Beijing, China, August 2018.

[3] L. Yang, X. Jin, and Q. Jiang, "Online handwritten signature verification based on the most stable feature and partition," Cluster Computing, vol. 22, pp. 1-11, 2018.

[4] H. H. Le and N. T. Do, "Offline handwritten signature verification using local and global features," Annals of Mathematics and Artificial Intelligence, vol. 75, no. 1-2, pp. 231-247, 2015.

[5] G. HafemannL, R. Sabourin, and L. S. Oliveira, "Writer-independent feature learning for offline signature verification using deep convolutional neural networks," in Proceedings of the 2016 International Joint Conference on Neural Networks (IJCNN), pp. 2576-2583, IEEE, Vancouver, Canada, July 2016.

[6] J. Hu, Z. Guo, Z. Fan, and Y. Chen, "Offline signature verification using local features and decision trees," International Journal of Pattern Recognition and Artificial Intelligence, vol. 31, no. 3, Article ID 1753001, 2017.

[7] M. Sharif, M. A. Khan, M. Faisal et al., "A framework for offline signature verification system: best features selection approach," Pattern Recognition Letters, vol. 139, 2018.

[8] N. Bouadjenek, H. Nemmour, and Y. Chibani, "Age, gender and handedness prediction from handwriting using gradient features," in Proceedings of the 2015 13th International Conference on Document Analysis and Recognition (ICDAR), pp. 1116-1120, IEEE, Tunis, Tunisia, August 2015.

[9] R. Kaur and M. P. Choudhary, "Handwritten signature verification based on surf features using HMM," International Journal of Computer Science Trends and Technology (IJCST), vol. 3, no. 1, pp. 187-195, 2015.

[10] E. N. Zois, L. Alewijnse, and G. Economou, "Offline signature verification and quality characterization using poset-oriented grid features," Pattern Recognition, vol. 54, pp. 162-177, 2016.

[11] O. A. Rosso, R. Ospina, and A. C. Frery, "Classification and verification of handwritten signatures with time causal information theory quantifiers," PLoS One, vol. 11, no. 12, Article ID e0166868, 2016.

[12] N. Li, J. Liu, Q. Li et al., "Online signature verification based on biometric features," in Proceedings of the 2016 49th Hawaii International Conference on System Sciences (HICSS), pp. 5527-5534, IEEE, Koloa, HI, USA, January 2016.

[13] C.. Z. G. TaşkiranM, "Offline signature identification via HOG features and artificial neural networks," in Proceedings of the 2017 IEEE 15th International Symposium on Applied Machine Intelligence and Informatics (SAMI), IEEE, Herlany, Slovakia, January 2017.

[14] N. Bouadjenek, H. Nemmour, and Y. Chibani, "Fuzzy integrals for combining multiple SVM and histogram features for writer's gender prediction," IET Biometrics, vol. 6, no. 6, pp. 429-437, 2017.

[15] B. S. Thakare and H. R. Deshmukh, "A combined feature extraction model using SIFT and LBP for offline signature verification system," in Proceedings of the 2018 3rd
International Conference for Convergence in Technology (I2CT), pp. 1-7, IEEE, Pune, India, April 2018.

[16] S. Shariatmadari, S. Al-maadeed, Y. Akbari et al., "Off-line Persian signature verification using wavelet-based fractal dimension and one-class Gaussian process," in Proceedings of the 2018 NASA/ESA Conference on Adaptive Hardware and Systems (AHS), pp. 168-173, IEEE, EdinBurgh, UK, August 2018.

[17] Y. Guerbai, Y. Chibani, and B. Hadjadji, "The effective use of the one-class SVM classifier for handwritten signature verification based on writer-independent parameters," Pattern Recognition, vol. 48, no. 1, pp. 103-113, 2015.

[18] H. Hezil, R. Djemili, and H. Bourouba, "Signature recognition using binary features and KNN," International Journal of Biometrics, vol. 10, no. 1, pp. 1-15, 2018.

[19] P. Maji, S. Chatterjee, S. Chakraborty et al., "Effect of Euler number as a feature in gender recognition system from offline handwritten signature using neural networks," in Proceedings of the 2015 2nd International Conference on Computing for Sustainable Global Development (INDIACom), pp. 1869-1873, IEEE, New Delhi, India, March 2015.

[20] S. Qiu, J. Luo, S. Yang, M. Zhang, and W. Zhang, "A moving target extraction algorithm based on the fusion of infrared and visible images," Infrared Physics \& Technology, vol. 98, pp. 285-291, 2019.

[21] J. Coetzer, B. M. Herbst, and J. A. du Preez, "Offline signature verification using the discrete radon transform and a hidden Markov model," EURASIP Journal on Applied Signal Processing, vol. 2004, pp. 559-571, 2004.

[22] C. Boufenar, M. Batouche, and M. Schoenauer, "An artificial immune system for offline isolated handwritten Arabic character recognition," Evolving Systems, vol. 9, no. 1, pp. 25-41, 2018.

[23] M. Diaz, M. A. Ferrer, G. Pirlo et al., "Off-line signature stability by optical flow: feasibility study of predicting the verifier performance," in Proceedings of the 2015 International Carnahan Conference on Security Technology (ICCST), pp. 341-345, IEEE, Taipei, Taiwan, September 2015.

[24] C. I. Gonzalez, P. Melin, J. R. Castro, O. Castillo, and O. Mendoza, "Optimization of interval type-2 fuzzy systems for image edge detection," Applied Soft Computing, vol. 47, pp. 631-643, 2016.

[25] J. Shi, J. Wu, M. Anisetti, E. Damiani, and G. Jeon, "An interval type-2 fuzzy active contour model for auroral oval segmentation," Soft Computing, vol. 21, no. 9, pp. 2325-2345, 2017.

[26] S. Dhar and M. K. Kundu, "A novel method for image thresholding using interval type-2 fuzzy set and Bat algorithm," Applied Soft Computing, vol. 63, pp. 154-166, 2018.

[27] P. Burillo and H. Bustince, "Entropy on intuitionistic fuzzy sets and on interval-valued fuzzy sets," Fuzzy Sets and Systems, vol. 78, no. 3, pp. 305-316, 1996.

[28] W. Zeng and H. Li, "Relationship between similarity measure and entropy of interval valued fuzzy sets," Fuzzy Sets and Systems, vol. 157, no. 11, pp. 1477-1484, 2006.

[29] H. Daher, V. Eglin, S. Bres, and N. Vincents, “A new approach for centerline extraction in handwritten strokes: an application to the constitution of a code book," in Proceedings of the Ninth IAPR International Workshop onDocument Analysis Systems, pp. 425-432, Boston, MA, USA, June 2010.

[30] J. Ma, F. Lin, S. Wesarg et al., "A novel Bayesian model incorporating deep neural network and statistical shape model for Pancreas segmentation," in Proceedings of the International Conference on Medical Image Computing and 
Computer-Assisted Intervention, pp. 480-487, Springer, Granada, Spain, September 2018.

[31] Z. M. Yaseen, R. C. Deo, A. Hilal et al., "Predicting compressive strength of lightweight foamed concrete using extreme learning machine model," Advances in Engineering Software, vol. 115, pp. 112-125, 2018.

[32] L. Gan, J. Xia, P. Du, and J. Chanussot, "Multiple feature Kernel sparse representation classifier for hyperspectral imagery," IEEE Transactions on Geoscience and Remote Sensing, vol. 56, no. 9, pp. 5343-5356, 2018.

[33] https://cedar.buffalo.edu/NIJ/data/.

[34] M. K. Sharma and V. P. Dhaka, "Pixel plot and trace based segmentation method for bilingual handwritten scripts using feedforward neural network," Neural Computing and Applications, vol. 27, no. 7, pp. 1817-1829, 2016. 